Научная статья

УДК $338.1(311.2)$

DOI: $10.17213 / 2075-2067-2021-6-206-218$

\title{
ИЗМЕРЕНИЕ «ЦИФРОВОЙ» ЭКОНОМИКИ И ПСЕВДОЭФФЕКТ ТЕХНОЛОГИЧЕСКОГО ДУАЛИЗМА ${ }^{1}$
}

\author{
Олег Сергеевич Сухарев
}

\author{
Институт экономики РАН, Москва, Россия \\ o_sukharev@list.ru, ORCID:0000-0002-3436-7703, Researcher ID: C-3767-2018
}

Аннотация. Цель исследования. Управляемое распространение цифровых технологий стало атрибутом экономической политики во многих странах, войдя в качестве базовой задачи в доктрину «Индустрия 4.0». Решение этой задачи требует учета исходного состояния технологического базиса экономики, готовности всех звеньев экономики к освоению новых технологий. Эффект технологического дуализма может оказывать сдерживающее влияние на распространение цицрровых и иных технологий, что требует отдельного изучения. Целью исследования выступает построение методики оценки распространения ичифровых технологий и идентификачии эффекта технологического дуализма, который для российской экономики приобретает специфическое содержание.

Методологию исследования составляет теория технологического развития и эффективности функционирования объектов экономики, подходы в области экономических измерений и формирования алгоритма и методики оценки релевантных параметров.

Результат исследования состоит в том, что в российской экономике выявлен псевдоэффект технологического дуализма на основе анализа динамики основного капитала и перемещения труда между экономическими секторами. Его возникновение может блокировать технологическое развитие, не препятствуя при этом ходу прочесса распространения циифровых технологий. Обоснованы два базовых варианта распространения ичифровых технологий и предложена методика оценки масштаба их распространения в экономике с оценкой эффективности ввода, а также методика идентификации технологического дуализма. Показано, что отсутствие роста производительности при расширении компьютерной техники и сетевых технологий не является парадоксом, а составляет вполне закономерный итог, обусловленный не только капитальным вложением на внедрение этих технологий, но и необходимостью обучения персонала, а также исходным масштабом рынка продуктов, создаваемых объектами, осуществляющими ввод ицирровых технологий.

Перспектива исследования. Для изучения изменений структуры технологической динамики на перспективу предложен инструмент технологической карты и портфеля, требующие расширенного статистического учета технологий и описывающих их параметров, позволяющие расширить представление о влиянии технологий на экономическое развитие и эффекте технологического дуализма.

Ключевые слова: методология измерений, цифрровые технологии, «парадокс» производительности, псевдоэффект «технологического дуализма»

(C) Сухарев О.С., 2021

1 Статья подготовлена на основе пленарного авторского доклада «Распространение цифровых технологий: оценка влияния» на Международной научно-практической конференции «Управление культурными различиями и цифровизация как векторы устойчивого развития высшего образования: международный опыт и особенности Центральной Азии и России», проводимой ЮРГПУ (НПИ) 15 октября 2021 г. 
Для цитирования: Сухарев О.С. Измерение «цифровой» экономики и псевдоэффект технологического дуализма // Вестник Южно-Российского государственного технического университета. Серия: Социально-экономические науки. 2021. T. 14, № 6. C. 206-218. http:// dx.doi.org/10.17213/2075-2067-2021-6-206-218.

Original article

\title{
MEASURING THE «DIGITAL» ECONOMY AND PSEUDO EFFECT OF TECHNOLOGICAL DUALISM
}

\author{
Oleg S. Sukharev
}

\section{Institute of Economics, Russian Academy of Sciences, Moscow, Russia o_sukharev@list.ru,ORCID:0000-0002-3436-7703, Researcher ID: C-3767-2018}

\begin{abstract}
The purpose of the study. The controlled diffusion of digital technologies has become an attribute of economic policy in many countries, entering as a basic task in the doctrine of "Industry 4.0». Solving this problem requires taking into account the initial state of the technological basis of the economy, the readiness of all parts of the economy to master new technologies. The effect of technological dualism can have a deterrent effect on the spread of digital and other technologies, which requires a separate study. The purpose of the study is to build a methodology for assessing the spread of digital technologies and identifying the effect of technological dualism, which acquires a specific content for the Russian economy.
\end{abstract}

The research methodology is the theory of technological development and the efficiency of functioning of economic objects, approaches in the field of economic measurements and the formation of an algorithm for assessing the relevant parameters.

The result of the study is that a pseudo-effect of technological dualism has been identified in the Russian economy, based on an analysis of the dynamics of fixed capital and the movement of labor between economic sectors. Its occurrence can block technological development without interfering with the course of the digital diffusion process. Two basic options for the dissemination of digital technologies have been substantiated, and a methodology for assessing the scale of their spread in the economy, with an assessment of the efficiency of input, as well as a method for identifying technological dualism has been proposed. It is shown that the lack of productivity growth with the expansion of computer technology and network technologies is not a paradox, but is a completely natural result, due not only to capital investments in the implementation of these technologies, but also to the need for personnel training, as well as the initial scale of the market for products created by facilities that carry out input of digital technologies.

The prospect of the study. To study the changes in the structure of technological dynamics for perspective, a tool of a technological map and a portfolio is proposed, which require an extended statistical accounting of technologies and their parameters, which make it possible to expand the understanding of the impact of technologies on economic development and the effect of technological dualism.

Key words: measurement methodology, digital technologies, productivity «paradox», pseudoeffect of «technological dualism»

For citation: Sukharev O.S. Measuring the «digital» economy and pseudo effect of technological dualism // Bulletin of the South Russian State Technical University. Series: Socio-economic Sciences. 2021; 14(6): 206-218. (In Russ.). http://dx.doi.org/10.17213/2075-2067-2021-6-206-218. 
Введение. Рассматривая экономический рост и развитие с ориентацией на различные подходы и модели неоклассического или иного типа [1-2; 10-11], можно отметить, что своеобразным объединяющим их началом выступает проблема измерения. Для сравнительных исследований по странам ее просто никак нельзя обойти. Причем критика различных показателей, например, ВВП, заставляющая экономистов искать иные способы оценки развития, тем не менее, несмотря на разработку этих методов, не снимает проблемы различных способов измерения таких социальных явлений, как неравенство, бедность ${ }^{2}$ [6-7]. Виды деятельности и экономические секторы измеряются обычно весьма просто - по величине создаваемой добавленной стоимости, числу занятых людей, фондам, активам и т.д. Однако, если сектор выделяется методом агрегации как трансакционный сектор, включающий все виды трансакционной деятельности по всем сферам или «цифровой экономики», представляющей работу цифровых технологий, то в таком случае уже само выделение является своеобразной моделью, поэтому здесь присутствуют эмпирические замеры. Но в реальности такого сектора как объекта нет, он построен исследователем, включая и способы измерения, и показатели оценки функционирования. Причем этот прием используется весьма часто в современных экономических исследованиях и органах статистического учета. В частности, «экономика знаний» согласно Евростату [8] измеряется числом людей с определенным уровнем образования, превышающим установленную норму (33\% от всего состава занятого персонала). Тем самым объект относится к экономике знаний, хотя может совсем не иметь отношения ни к производству знаний, ни к их значимому тиражированию, обучению. Аналогичная, если не более серьезная, проблема касается «цифровой экономики», поскольку измерить ее по занятому в работе с цифровыми технологиями персоналу будет еще более проблематично, так как технологии высвобождают персонал.
В принципе, термин «цифровая экономика» выглядит не менее надуманным, нежели «наноэкономика» или «ноономика», а также ставшие модными сегодня так называемые «экосистемы». Само по себе применение этого термина не проясняет наблюдаемую экономическую реальность, поскольку как таковой цифровой экономики не существует, а только усложняет ее восприятие, создавая ложное противопоставление между системным содержанием экономики [5] и масштабом применяемых цифровых технологий, закономерно вытесняющих аналоговые технологии. Казалось бы, наименование «цифровая экономика» сразу обозначает сегмент хозяйства и необходимость его измерения, которую можно реализовать, выделяя долю цифровых технологий в общем объеме вновь вводимых технологий, либо долю добавленной стоимости, производимой при участии цифровых технологий, либо долю занятых в обслуживании цифровых технологий относительно общей величины занятого населения. Однако приведенные варианты измерения дают три количественные оценки, которые могут характеризовать разный охват распространения цифровых технологий.

Измерение как метод является в экономической науке основополагающим. От него зависит оценка не только явления или объекта, но и происходящих изменений, а также уровень обоснования необходимых управленческих воздействий с тем, чтобы изменить состояние объекта или ход процесса, содержание наблюдаемого явления.

Например, можно измерять размер государственного сектора по доле добавленной стоимости в общей ее величине в экономике. Однако, если размер и состав этого сектора законодательно не установлен, то измерение становится нормативно-фривольным, зависящим то того, как этот сектор определяет субъект, осуществляющий измерение. Если компании с государственным акционерным участием, скажем, от 30 до 49\% акций, отнести к государственному сектору, то тогда возникает проблема: как отнести к государс-

2 Стиглицу Дж., измерившему доходы $1 \%$ населения США и иных 90\% населения, удалось показать, что реальные доходы $90 \%$ населения в отличие от $1 \%$ практически не росли на длительном интервале времени, в то время как 1\% несказанно разбогател. Применение стандартных методов в виде коэффициента Джини, показателя фондов, либо индекса Э. Аткинсона не даёт такой оценки. Разложение по доходным группам может предполагать процедуру, похожую на применение индекса социального неравенства А. Тейла. 
твенному сектору создаваемую такими компаниями добавленную стоимость - в таком же соотношении или нет? Однако указанное выделение касается пакета акций, что совсем не означает влияния на создаваемую добавленную стоимость. Обратим внимание, что критерий «выделение» является главной детерминантой. Выделим государственный сектор, и, посчитав добавленную стоимость, можем получить оценку, скажем, в 70\% добавленной стоимости, приходящейся на этот сектор. Тогда, если эта цифра справедлива для России, возникнет вопрос относительно того, что именно такая же доля добавленной стоимости приходится на услуги, которые в подавляющем объеме создаются частным сектором (торговля занимает до $27 \%$ валовой добавленной стоимости для России). Следовательно, если границы сектора не определены, не ясен его состав, масштаб, правом не отрегулировано отнесение организаций к нему и не ясна методика оценки разбивки созданной добавленной стоимости, когда компанией владеют и частники, и государство, то измерение такого сектора может привести к неверным оценкам его чрезмерно крупного масштаба. При этом могут производиться оценки его низкой эффективности. Вместе с тем, если сектор имеет малые активы и дает высокую величину добавленной стоимости, нежели частный сектор, имеющий большие активы, ему принадлежавшие, но дающий меньшую величину добавленной стоимости, то вопрос об эффективности снимается автоматически, причем отнюдь не в пользу частного сектора.

Таким образом, измерение в экономике в значительной степени зависит от критерия и от метода получения данных, определения границ измеряемого объекта [4; 8-9]. Если цифровая экономика - это набор цифровых технологий, включая их обслуживание, то такой сегмент может измеряться по числу технологий, создаваемой добавленной стоимости, занятому персоналу, масштабу обслуживающей инфраструктуры и пр. Поскольку здесь ключевое значение имеет технология, то важно не столько, что она цифровая, а как именно она воплощена в оборудовании и приборах, компьютерах и коммуникации, т.е. к какому быстродействию и обработке информации она приводит. Иными словами, параметры качества становятся важнейшим условием распространения цифровых технологий, причем восприятие их всеми элементами экономики играет важную роль, как и состояние производственных систем, инфраструктуры [13-14].

Программы развития сильно влияют на распространение новых технологий, но время как ресурс и оценки с позиции учета именно этого ресурса при рассмотрении различных вариантов помощи развитию и управления развитием становятся ключевыми позициями в «измерении развития» [16].

Как видим, проблема измерения «цифровой экономики» касается, как минимум, двух центральных моментов: распространения цифровых технологий и оценки эффективности их внедрения и дальнейшего использования. В связи со сказанным целью настоящего исследования выступает разработка авторской методики измерения распространения цифровых технологий, а также оценки их эффективности и идентификации эффекта технологического дуализма, включая его псевдовариант.

Для ее достижения потребуется разрешить некоторые методологические вопросы измерения технологического развития, а также рассмотреть влияние «парадокса производительности» и российский вариант технологического дуализма, который в ранних работах автора был обозначен псевдоэффектом [9].

\section{1. Методология измерения технологи-} ческого развития. Измерение технологического развития выступает наиболее сложной областью измерений, поскольку статистическая информация, учет данных объективно не обладают той полнотой, как по иным сферам и показателям общего макроэкономического свойства. Кроме того, технологические факторы подлежат включению в модели роста и развития, причем от математической формализации во многом зависит правдоподобие получаемых оценок и выводов для экономической политики [3].

Для измерения технологического развития могут применяться абсолютные показатели, характеризующие технологические инновации, масштаб применения новых технологий, обладание высокими технологиями. Кроме них используются показатели, такие 
как уровень технологичности, не имеющие однозначного измерения в каких-либо единицах, подобно технологичности в технических системах, где она выступает комплексной характеристикой условий производства изделия, эксплуатации и ремонта. В экономике технологичность означает применение способа получения такого же результата с меньшими издержками либо большего результата с теми же издержками, достигается за счет ввода новых технологий или усовершенствования уже используемых технологий. Исходный уровень технологичности экономики сильно влияет на ход технологического обновления, инвестиции в новые технологии, внедрение цифровых технологий [9].

Проблема измерения технологичности предполагает введение показателя уровня технологичности либо использование набора показателей, характеризующих общую технологичность. Росстат выделяет высоко-, средне- и низкотехнологичные виды деятельности, причем средняя группа имеет еще два выделения - это низко-, высоко-среднетехнологичные виды деятельности. Однако, выделение этих видов осуществляется по доле затрат на НИОКР в общей величине производства или добавленной стоимости видов деятельности [8].

Технологическое развитие охватывает довольно продолжительный интервал времени, в связи с чем оценивать его можно, не только используя названные показатели, но и прибегая к модельным построениям, в частности, выделяя технологические уклады и их преобладание в экономике. Структура новых укладов будет обеспечивать технологическую новизну, а преобладание старых технологических переделов при доминировании устаревших укладов - закреплять имеющиеся технологические возможности. Уровень технологического развития сегодня измеряется официально по типам высокотехнологичных видов деятельности, причем выделяются низко-, средне- и высокотехнологичные виды экономической деятельности. Отнесение к типу осуществляется по величине либо доле расходов на НИОКР. Необходимо отметить, что в таком случае априори предполагается связь между величиной расходов на НИОКР и уровнем технологичности. В действительности такой связи может не быть, посколь- ку небольшие затраты на НИОКР могут создать технологический прорыв, а значительные затраты ни к чему не привести. Пример российской экономики, когда в гражданских производствах не наблюдается увеличение технологичности, а оборонный сектор делает технологический рывок, несмотря на то что военные НИОКР на порядок меньше финансируются, нежели у зарубежных конкурентов, является весьма ярким подтверждением сказанному. Причина в том, что прямая связь между затратами на НИОКР и уровнем технологичности, видимо, не прослеживается. Часть НИОКР в принципе не оканчивается появлением новой технологии, другая часть имеет отрицательные результаты, и только некая доля приведет к появлению каких-то технологий, которые потребуется внедрить в существующую на этот момент времени технологическую цепочку. Поэтому выстраивание системы измерений и оценок на этой основе приводит к существенным искажениям. Подобные неоднозначности в измерении технологического развития подтверждают необходимость весьма взвешенного подхода в области оценки создаваемых и применяемых технологий. Это относится и к измерению производительности, в частности высокопроизводительных рабочих мест, которые нельзя оценивать по превышению заработной платы некоего установленного норматива, как и по добавленной стоимости. Причина в том, что высокая производительность обеспечивается уровнем автоматизации рабочего места и электронизации (цифровизации), а не оценкой труда или рыночной оценкой создаваемого блага или даже общей эффективностью хозяйствования - умением создавать высокую добавленную стоимость.

Как видим, методологические трудности измерения технологического развития пронизывают современную систему учета. Они настолько занчимы, что сильно детерминируют затем принимаемые решения и формируемую стратегию научно-технического развития страны. Ввод технологий требует оценки уровня их охвата плюс эффективности внедрения. Остановимся далее на этих аспектах, подчеркивая влияние эффекта технологического дуализма с российской спецификой и объясняя отсутствие парадокса в необнаруживаемом росте производительности. 
2. Распространение цифровых технологий, «парадокс» производительности и псевдоэффект «технологического дуализма». Сами по себе новые технологии, приводящие к автоматизации производства, программируя решения в этой области, не гарантируют получения выгод от применения. Причина состоит в «парадоксе» производительности [15], определяемом эффективностью внедрения и дальнейшей эксплуатации новых технологий, а также эффектами технологического замещения и дополнения, которые при этом возникают. В итоге технологическая революция сопровождается замедлением роста производительности [17]. Так, по данным Росстата, в нашей стране в период 2012-2018 гг. рост производительности труда по измеряемому индексу понижался, например, в секторе добычи полезных ископаемых с 101,5 до $100,3 \%$, в обработке - co 105,8 до 101,8\%, в строительстве со 102,4 до $100,7 \%{ }^{3}$. Число персональных компьютеров, серверов, локальных сетей, облачных сервисов в среднем возросло, не поддерживая темпа роста производительности [9], наоборот, происходило снижение динамики производительности. Безусловно, компьютеризация и распространение цифровых технологий происходили одновременно с активизацией целого набора тормозящих производительность факторов, связанных с мотивами, снижением реальных доходов населения, ростом издержек, отсталостью общей производственно-аппаратной базы и прочими видами технологий, конфликтующими с данным вводом либо воспринимающими его параллельно их применению и т.д. Иными словами, компьютеризация осуществлялась сама собой, а по другим элементам технологического базиса равнозначного замещения не происходило, так как оно требует высоких капитальных затрат. Даже при осуществлении таких капитальных вложений первый эффект может состоять в торможении роста производительности, так как значение приобретает оценка производительности сразу после ввода или с течением времени, когда учтен лаг адаптации к новым технологиям.

Однако, на взгляд автора, обозначенные процессы не составляют никакого парадок$\mathrm{ca}^{4}$, особенно если принимать во внимание цифровые технологии, представленные в виде компьютерной техники. Причина, конечно, не столько в методологических трудностях измерения производительности [12], сколько в специфическом встраивании указанных технологий в общий технологический контур экономики. Нет однозначной гарантии даже в экономии издержек, хотя ряд функций выполняется быстрее и эффективнее, включая и поиск информации. Однако количество информации и задачи по обслуживанию техники и созданию программного обеспечения резко увеличиваются не только в объеме, но и в сложности. Суммируя, отметим, что помимо распространения какого-то типа технологий важнейшим параметром выступает эффективность их применения, которая диктует целесообразность внедрения, т.е. влияет на ход распространения. Тем самым и распространение, и эффективность выступают детерминирующими параметрами при рассмотрении процесса технологического обновления и развития.

Цифровые технологии, замещающие аналоговые технологии, распространяясь, не дают значимого прироста производительности по целому ряду причин, причем не только сугубо технологических. Сгруппируем их.

Во-первых, высокие издержки ввода новой технологии и ее обслуживания, зависимые от уровня развития отдельных отраслей, в частности микроэлектроники и др.

Во-вторых, издержки обучения кадров новой технологии, а также конфликта с устаревающими технологиями, зависимости от текущего состояния производств и видов труда, когда масштаб развития какой-то сферы и ее рынков предопределяет уровень распространения цифровых технологий и эффективность их ввода.

3 Источник: по данным Росстата. URL: https:/www.gks.ru/folder/11186.

4 Тот факт, что автоматизация в 1960-1970-е гг. не всегда давала рост производительности и конфликтовала с текущими задачами развития производства, признавался многими экономистами того периода [4], утверждавшими, что нельзя измерять, в частности производительность, по объёму изделий, изготавливаемых в единицу времени, хотя именно такое измерение будет отражать полезный эффект автоматизации производства. 
В-третьих, наличие псевдоэффекта технологического дуализма, при котором при вводе капиталоинтенсивных технологий ${ }^{5}$ не происходит высвобождения труда, направляющегося в трудоинтенсивные секторы экономики по причине, что труд высвобождается в силу структурных диспропорций и неэффективности, перемещаясь из капиталоинтенсивных в трудоинтенсивные секторы. Это приводит к тому, что в капиталоинтенсивных секторах затрудняется ввод новых технологий, экономящих труд. В трудоинтенсивных видах деятельности блокируется в принципе применение технологий, высвобождающих труд. Учитывая, что компьютерная техника в среднем значительно дешевле иных средств производства, а ее применение требует пусть и подготовленного, но труда, можно сказать, что поэтому ее не отнесешь сугубо к капиталоинтенсивной технологии, высвобождающей труд во всех ситуациях. Компьютеры требуют операторов и обслуживающего персонала, разработчиков программного обеспечения, ремонтников и т.д. Применение их для конкретной организации может быть новым, но в общем смысле таковым не быть.

Ввод новых технологий может быть для данной организации, но не для рынка, в связи с чем оценка уровня технологических инноваций, если так трактуется эффект новизны, будет принимать во внимание тиражирование технологий, но не чистый эффект их распространения. Поэтому измерение уровня технологичности по величине технологичес- ких инноваций следует делать с оговорками на то, как именно оцениваются эти инновации. Дуализм технологий в том, что применяя экономящие труд технологии, высвобождая труд, он находит применение в иных видах деятельности, что снижает технологическую безработицу в ходе технологического обновления экономики. При этом все-таки общий технологический уровень повышается. Если происходит перемещение труда между секторами, но не по причине ввода капиталоинтенсивных технологий, а в силу структурных диспропорций (рентабельность - риск ведения деятельности сильно отличаются, порождая градиент перемещения ресурсов между видами деятельности), то технологический уровень не повышается, иногда снижается. Эффект технологического дуализма в этом случае расходится с классической трактовкой, поэтому его можно обозначить как «псевдоэффект» ${ }^{6}$.

В таблице 1 приводится расчет, показывающий, как идет процесс увеличения основных фондов в обработке и трансакционных с сырьевыми секторами, а также отток кадров из обработки, но значимо большее увеличение труда в трансакционных и сырьевых секторах, нежели отток из обработки. При этом показатели производительности и технологичности не возрастают, подтверждая наличие псевдоэффекта технологического дуализма.

Для российской экономики наличие такого псевдоэффекта, с одной стороны, бло-

5 Речь идёт о технологиях двух больших типов: капиталоинтенсивных и трудоинтенсивных. Капиталоинтенсивные высвобождают труд (трудосбережение). Трудоинтенсивные высвобождают капитал. При технологическом дуализме секторы с трудоинтенсивными технологиями снижают технологическую безработицу, принимая высвобождаемый труд. Кстати, обратного эффекта, когда трудоинтенсивные виды деятельности высвобождают капитал, не наблюдается. Этот капитал не принимается капиталоинтенсивными секторами. Обычно обратный эффект может быть выражен в размещении инвестиций между секторами. Технологически дуализм состоит в том, что оба типа технологий сосуществуют, причем не вытесняя друг друга. Возникает своеобразное равновесие, но технологическое обновление тормозится. При перемещении труда из капиталоинтенсивных видов деятельности в область трудоинтенсивных технологий не под влиянием процесса ввода трудосберегающих технологий возникает особый эффект «технологического дуализма» - псевдоэффект. Технологическое обновление в системном ракурсе тормозится.

6 «Псевдо» в значении «как бы», т.е. отличающийся от стандартного представления эффекта технологического дуализма. Наличие псевдоэффекта в отличие от стандартного эффекта тормозит технологическое обновление, так как препятствует вводу капиталоинтенсивных технологий благодаря утечке труда, происходящей вне связи с ними. В трудоинтенсивных секторах данный труд находит место применения, что может расширить возможность для цифровизации такого труда, поскольку цифровые технологии по широкому классу объектов можно считать как слабо капиталоинтенсивные технологии. В классическом эффекте технологическое обновление также может само тормозиться, ибо сосуществуют два типа конкурирующих технологий (капитало- и трудоинтенсивные), возникает равновесие между ними. При псевдоэффекте сразу блокируется возможность существенного технологического обновления, которое реализуется только за счёт локальных действий. При этом инфраструктурные возможности цифровых технологий могут расширяться в рамках трудоинтенсивных секторов, которые подлежат цифровизации. 
Оценка псевдоэффекта технологического дуализма в РФ, 2006-2019 гг. ${ }^{7}$ Evaluation of the pseudo-effect of technological dualism in the Russian Federation, 2006-2019

\begin{tabular}{|l|c|c|c|c|}
\hline \multirow{2}{*}{ Экономический сектор } & \multicolumn{2}{|c|}{ Основные фонды } & \multicolumn{2}{c|}{ Занятые } \\
\cline { 2 - 5 } & млн руб. в ценах 2005 г. & $\%$ & тыс. чел. & $\%$ \\
\hline Обрабатывающий & 3555837 & 17,29 & -1461 & $-2,09$ \\
\hline Трансакционно-сырьевой & 23149007 & 140,51 & 4886 & 6,9 \\
\hline
\end{tabular}

кирует распространение капиталоинтенсивных технологий в базовых отраслях, с другой стороны, вполне обеспечит цифровизацию в трудоинтенсивных видах деятельности, принимающих труд. При этом показатели производительности технологичности и эффективности могут не изменяться, что и наблюдается на практике. Тем самым наличие псевдоэффекта не влияет на торможение распространения цифровых технологий, но и не позволяет достичь значимых показателей по уровню технологичности и производительности.

Рассматривая технологический дуализм, а также псевдоэффект, можно сказать, что существует важная задача выделения - идентификации того, насколько он распространен в экономике, то есть степени глубины и силы влияния. Для этой цели предложим следующую методику, представленную ниже основными шагами:

- установить виды деятельности (труда) с капиталоинтенсивными и трудоинтенсивными технологиями, построить технологическую карту труда, на основе которой сформировать «технологический портфель» ${ }^{8}$;

- осуществить измерение движения рабочей силы из видов деятельности с капиталоинтенсивными в область с трудоинтенсивными технологиями, определить причины и последствия для кадрового состава в видах деятельности;

- рассчитать величину технологической безработицы, возникающую по причине ввода капиталоинтенсивных технологий, т.е. в ходе процесса технологического обновления;
- выявить динамику ввода капиталои трудоинтенсивных технологий посредством определения разрыва между разработанными и внедренными технологиями, а также передовыми технологиями для каждого вида деятельности или сектора;

- оценить разновидность (стандартный или псевдо) эффекта технологического дуализма, уровень влияния на экономическую динамику, показатели: эффективность, производительность и технологичность.

Важна оценка силы блокирования дальнейшего технологического обновления и развития экономики и ее секторов.

Технологическая карта представляет собой распределение технологий по видам деятельности, секторам, отраслям, агентам $(1 \ldots i \ldots N)$ на какой-то момент времени. Тем самым она отражает технологическую статику (см. таблицу 2).

Технологический портфель отражает то, как распределены технологии в рамках выделенных экономических объектов, показывая уровень технологической концентрации в своих границах. Например, технологический портфель покажет, что большая часть технологических инноваций сосредоточена в обрабатывающих секторах, промышленности, а не торговле или недвижимости. Технологии информационной обработки, наоборот, будут сконцентрированы в секторах услуг, финансах, консалтинге. Портфель показывает связь технологий и подчинен задаче отражения изменения этой связи, демонстрируя технологическую динамику. Каждый

7 Источник: рассчитано автором по данным Росстат. URL: https://rosstat.gov.ru/labour_force, https://rosstat. gov.ru/free_doc/new_site/business/osnfond/nal_ved2.htm.

8 Полезно будет выделить в каждом виде деятельности агентов (фирмы), относимые к новаторам, консерваторам и имитаторам. 
элемент портфеля имеет свои параметры, характеризующие эффективность, величину инвестиций, риска и т.д. Следовательно, можно использовать общие подходы в рамках портфельного выбора, адаптируя их к выбору технологическому, с оговорками на содержательную сторону технологических изменений, обусловленную разнородностью технологий как объектов.

Как следует из таблицы 2, на какой-то момент времени объекты - первый, $i$-й и $N$-й, в качестве которых могут быть предприятия, регионы или даже страны, - обла- дают различным набором применяемых технологий из полного имеющегося их набора. В другой момент времени технологическая карта отразит иную картину. Технологический портфель покажет, как распределены ресурсы по этой технологической карте, т.е. какую их величину забирает реализация каждой из применяемых технологий. Фиксация двух состояний на каком-то интервале времени позволит утверждать, уменьшились или увеличились технологические возможности рассматриваемых объектов и за счет каких решений.

Таблица 2

Table 2

Технологическая карта («+» присутствует, «-» отсутствует технология) ${ }^{9}$ Technological map («+» is present, «-» there is no technology)

\begin{tabular}{|l|c|c|c|}
\hline \multicolumn{1}{|c|}{ Технические процессы (технологии) } & \multicolumn{3}{c|}{ Объект } \\
\cline { 2 - 4 } & $1-$ й & i-й & N \\
\hline Литье под давлением & + & - & - \\
\hline Точное литье & + & - & - \\
\hline Литье в землю & - & - & - \\
\hline Литье в кокиль & - & - & - \\
\hline Холодная высадка & - & + & - \\
\hline Холодная штамповка & + & + & + \\
\hline Кузнечные работы & - & - & - \\
\hline Механическая обработка на универсальных станках & + & - & + \\
\hline Обработка на токарных станках & + & + & - \\
\hline Обработка на станках с ЧПУ & + & - & + \\
\hline Гальванические & + & + & + \\
\hline Малярные & + & - & + \\
\hline Изготовление печатных плат & + & - & - \\
\hline Переработка пастмасс & - & + & - \\
\hline Изготовление жгутов & - & - & - \\
\hline Электромонтажные & + & - & - \\
\hline Деревообработки & - & - & - \\
\hline Термические & + & + & + \\
\hline Испытательные станции & + & + & - \\
\hline Изготовление оснастки и инструмента & + & + & - \\
\hline Сварочные & + & - & + \\
\hline Конструкторско-технологические & & + \\
\hline
\end{tabular}

9 Составлено автором на основе полевых обследования предприятий. 
В таблице 2 приводятся сугубо производственные технологии, но каждая из них может предполагать применение цифровой технологии, поэтому возможно построение карты охвата цифровыми технологиями (технологической карты). Для этого там, где указаны плюсы, можно отразить добавочным плюсом применение цифровой технологии и по этому числу плюсов получить степень распространения цифровой технологии по трем сравниваемым объектам, просчитав долю «технологического охвата» (см. рисунок 1). По рисунку 1 можно видеть потенциал для цифровизации, определяемый площадью фигуры АБВГД, а также отличия в уровне цифровизации между сравниваемыми объектами. Конечно, требуется еще учитывать уровень самих цифровых технологий, применяются ли они в новых приборах и оборудовании либо просто замещают аналоговые технологии в применявшихся механизмах, автоматах, роботизированных системах.

Таким образом, обобщая, следует заметить, что методика измерения «цифровой экономики», на наш взгляд, должна строиться на основе двух базовых позиций.

Во-первых, необходима оценка распространения (охвата) цифровых технологий экономики в целом и отдельных ее объектов (регионов, секторов, отраслей, корпораций и т.д.). Данные оценки можно осуществлять по следующим направлениям: числу работ- ников, применяющих цифровые технологии; затратам труда на внедрение цифровых технологий; инвестиционным расходам и их окупаемости, включая экономию на себестоимости изделий; трудоемкости операций, выполняемых с применением цифровых технологий или без их использования. Здесь могут применяться следующие показатели:

- отношение числа занятых в области цифровых технологий (пользующихся цифровыми технологиями) к общей величине занятого персонала;

- отношение суммарных затрат на цифровизацию к общим затратам;

- отношение времени «цифрового труда» ко времени «нецифрового труда»;

- отношение времени «цифрового труда» ко всему времени по созданию одного и того же изделия, продукта, услуги;

- мощность (либо скорость процессоров и интернета) компьютеров, сетей, приходящаяся на одного работника;

- мощность компьютеров, сетей, приходящаяся на единицу потребляемой электрической энергии.

Также масштаб распространения может быть оценен по коэффициенту уровня цифровизации, представляющему собой отношение общих затрат на «цифровой труд» к такому объему затрат, когда все работы не были бы цифровизированы. Возможно определение уровня цифровизации по охвату

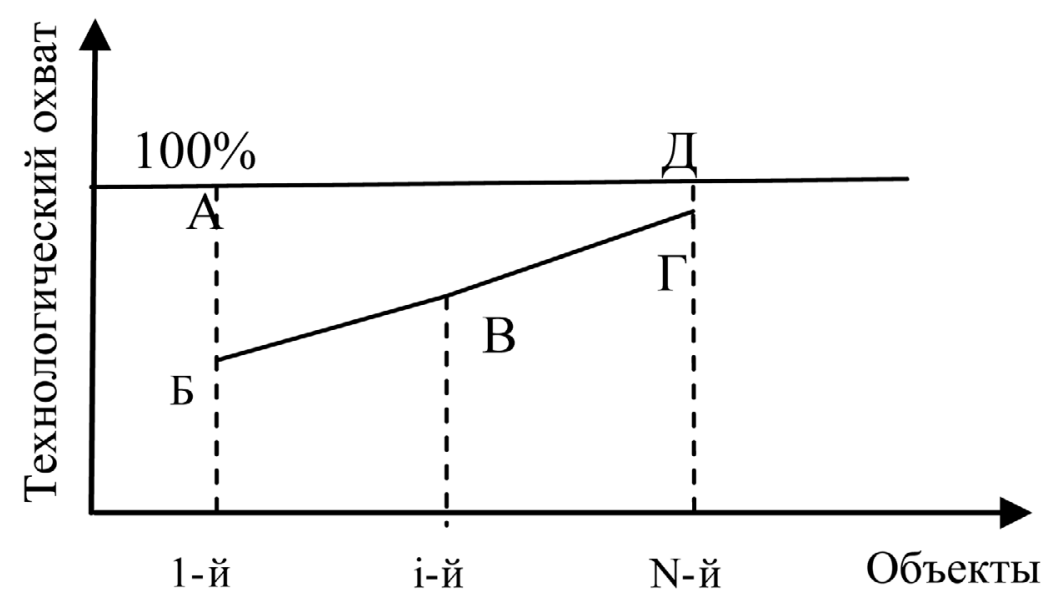

Рис. 1. Технологический охват по рассматриваемым объектам, показывающий распространение цифровых технологий в некий момент времени

Fig. 1. Technological coverage of the objects under consideration, showing the spread of digital technologies at a certain point in time 
работников как отношения числа использующих в своем труде полностью или частично цифровые технологии в общей величине занятого на объекте персонала.

Во-вторых, нужна оценка эффективности ввода цифровых технологий, которая может осуществляться стандартным способом согласно логике оценки инвестиционных расходов и окупаемости. Капитальные расходы на ввод цифровых технологий дисконтируются, но и оценка прибыли или дохода в течение последующих лет также должна дисконтироваться и сопоставляться с осуществленными затратами, причем включая и операционные расходы - текущие издержки поддержания работоспособности цифровой техники и устройств.

Конечно, оценка эффективности более сложна, нежели оценка распространения и охвата, поскольку необходимо измерять не только затраты, что поддается первичной оценке, как и их изменение, но и будущие выгоды, которые не являются столь однозначными. Для ряда объектов они зависят от рыночных перспектив создаваемых благ, что может не определяться напрямую уровнем цифровизации и степенью распространения цифровых технологий. Перспектива технологического обновления экономики не может быть точно очерчена без учета факторов, вызывающих «парадокс производительности», и что особо важно - эффекта технологического дуализма с российскими специфическими характеристиками (псевдоэффект).

Заключение. Успешная реализация программ и национальных проектов в российской экономике по ее цифровизации происходит на фоне возникновения псевдоэффекта технологического дуализма. За рассматриваемый период развития наблюдалось высвобождение кадров из капиталоинтенсивных секторов и размещение их в трудоинтенсивных видах деятельности. Однако причиной перемещения трудового ресурса был не ввод сберегающих труд технологий, а структурный межсекторный разрыв экономики и низкий уровень технологического обновления.

В связи с этим стереотипная политика стимулирования технологических инноваций, ввода новых технологий, включая цифровые, не приведет к кардинально иной дина- мике, хотя цифровизация инфраструктурных элементов может происходить вполне удачно. В стратегической перспективе потребуется иная модель технологического развития диффузии технологических инноваций, имеющих системные следствия и улучшения.

С этой целью полезными выступают две предложенные в настоящей статье методики - идентификации эффекта технологического дуализма и его российской версии (псевдоэффекта) и оценки распространения (охвата) цифровых технологий различных сегментов и экономики в целом, включая и измерение эффективности. Их необходимо применять при планировании ввода новых технологий, обеспечивающих электронизацию и автоматизацию производства и экономики. Следовательно, методы разработки национальных проектов могут включать обозначенные здесь предложения.

\section{Список источников}

1. Анчишкин А.И. Прогнозирование темпов и факторов экономического роста. М.: Макс-Пресс, 2003. 300 с.

2. Глазьев С.Ю. Битва за лидерство в XXI веке. Россия, США, Китай. Семь вариантов ближайшего будущего. М.: Книжный мир, 2017. 352 c.

3. Глазунова В.В. Моделирование технологических изменений: основные подходы / Анализ, моделирование и управление, развитие XV Всероссийской школы-симпозиума социально-экономических систем: сборник научных трудов. Симферополь: ИП Корниенко А.А., 2021. С. 111-114.

4. Кваша Я.Б. Избранные труды. В 3 т. Т. 1: Методологические основы статистического анализа. М.: Наука, 2003. С. 257-345.

5. Клейнер Г.Б. Системная экономика: шаги развития. М.: Издательский дом «Научная библиотека», 2021. $746 \mathrm{c.}$

6. Лившиц В.Н. Бедность и неравенство доходов населения в России и за рубежом. М.: ИЭ РАН, 2017. $52 \mathrm{c.}$

7. Стиглиц Дж. Цена неравенства. Чем расслоение общества грозит нашему будущему? М.: Эксмо, 2015. 512 с.

8. Сухарев О.С. Измерения в экономике: проблемы теории, моделирования и статистики / Анализ, моделирование и управление, 
развитие XV Всероссийской школы-симпозиума социально-экономических систем: сборник научных трудов. Симферополь: ИП Корниенко А. А., 2021. С. 395-400.

9. Сухарев О.С. Цифровизация и направления технологического обновления промышленности России / Journal of New Economy. T. 22. №1. C. 26-52.

10. Хелпман Э. Загадка экономического роста. М.: Издательство Института Е. Т. Гайдара. 2011. 240 c.

11. Aghion P., Howitt P. Some Thoughts on Capital Accumulation, Innovation, and Growth // Annals of Economics and Statistics. June 2017. №125/126. Pp. 57-78.

12. Brynjolfsson E. The Productivity Paradox of Information Technology: Review and Assessment. Cambridge, Massachusetts: MIT Sloan School of Management, 1994. 25 p.

13. Horvat D., Kroll H., Jäger A. Researching the Effects of Automation and Digitalization on Manufacturing Companies' Productivity in the Early Stage of Industry 4.0. Procedia Manufacturing, 2019. Vol. 39. Pp. 886-893.

14. Machado C.G., Winroth M., Carlsson D., Almström P., Hallin M. Industry 4.0 readiness in manufacturing companies: challenges and enablers towards increased digitalization. Procedia CIRP, 2019. Vol. 81. Pp. 1113-1118.

15. Solow R. We'd Better Watch Out, review of S. S. Cohen and J. Zysman, Manufacturing Matters: The Myth of the Post-Industrial Economy. New York Times Book Review, 1987. $36 \mathrm{p}$.

16. Tinbergen J. The Duration of Development. Journal of Evolutionary Economics, 1995. Vol. 5(3). Pp. 333-39.

17. Triplett J.E. The Solow productivity paradox: What do computers do to productivity? The Canadian Journal of Economics. 1999. Vol. 32. №2. Pp. 310-334.

\section{References}

1. Anchishkin A.I. Prognozirovanie tempov i faktorov jekonomicheskogo rosta [Forecasting the rates and factors of economic growth]. Moscow: Maks-Press, 2003. 300 p. (In Russ.).

2. Glaz'ev S. Ju. Bitva za liderstvo v XXI veke. Rossija, SShA, Kitaj. Sem' variantov blizhajshego budushhego [The battle for leadership in the 21st century. Russia, USA, China.
Seven options for the near future]. Moscow: Knizhnyj mir, 2017. 352 p. (In Russ.).

3. Glazunova V.V. Modelirovanie tehnologicheskih izmenenij: osnovnye podhody [Modeling technological changes: basic approaches]. Analiz, modelirovanie i upravlenie, razvitie XV Vserossijskoj shkoly-simpoziuma social'no-jekonomicheskih sistem: sbornik nauchnyh trudov [Analysis, modeling and management, development of the XV All-Russian school-symposium of socio-economic systems: collection of scientific papers]. Simferopol': IP Kornienko A.A., 2021. Pp. 111-114. (In Russ.).

4. Kvasha Ja. B. Izbrannye trudy. V 3 t. T. 1: Metodologicheskie osnovy statisticheskogo analiza [Selected Works. In 3 vol. Vol. 1: Methodological Foundations of Statistical Analysis]. Moscow: Nauka, 2003. Pp. 257-345. (In Russ.).

5. Klejner G. B. Sistemnaja jekonomika: shagi razvitija [System economics: development steps]. Moscow: Izdatel'skij dom «Nauchnaja biblioteka», 2021. 746 p. (In Russ.).

6. Livshic V.N. Bednost' i neravenstvo dohodov naselenija v Rossii i za rubezhom [Poverty and income inequality in Russia and abroad]. Moscow: IJe RAN, 2017. 52 p. (In Russ.).

7. Stiglic Dzh. Cena neravenstva. Chem rassloenie obshhestva grozit nashemu budushhemu? [The price of inequality. How does the stratification of society threaten our future?]. Moscow: Jeksmo, 2015. 512 p. (In Russ.).

8. Suharev O.S. Izmerenija v jekonomike: problemy teorii, modelirovanija i statistiki [Measurements in Economics: Problems of Theory, Modeling and Statistics]. Analiz, modelirovanie i upravlenie, razvitie XV Vserossijskoj shkoly-simpoziuma social'no-jekonomicheskih sistem: sbornik nauchnyh trudov [Analysis, Modeling and Management, Development of the XV All-Russian School-Symposium of Socio-Economic Systems: collection of scientific papers]. Simferopol': IP Kornienko A.A., 2021. Pp. 395-400. (In Russ.).

9. Suharev O.S. Cifrovizacija i napravlenija tehnologicheskogo obnovlenija promyshlennosti Rossii [Digitalization and directions of technological renewal of the Russian industry]. Journal of New Economy. 22(1): 26-52. (In Russ.).

10. Helpman Je. Zagadka jekonomicheskogo rosta [The Riddle of Economic Growth]. Moscow: Izdatel'stvo Instituta E.T. Gajdara. 2011. 240 p. (In Russ.). 
11. Aghion P., Howitt P. Some Thoughts on Capital Accumulation, Innovation, and Growth // Annals of Economics and Statistics. June 2017. №125/126. Pp. 57-78.

12. Brynjolfsson E. The Productivity Paradox of Information Technology: Review and Assessment. Cambridge, Massachusetts: MIT Sloan School of Management, 1994. 25 p.

13. Horvat D., Kroll H., Jäger A. Researching the Effects of Automation and Digitalization on Manufacturing Companies' Productivity in the Early Stage of Industry 4.0. Procedia Manufacturing, 2019. Vol. 39. Pp. 886-893.

14. Machado C.G., Winroth M., Carlsson D., Almström P., Hallin M. Industry 4.0 readiness in manufacturing companies: challenges and enablers towards increased digitalization. Procedia CIRP, 2019. Vol. 81. Pp. 1113-1118.

15. Solow R. We'd Better Watch Out, review of S.S. Cohen and J. Zysman, Manufacturing Matters: The Myth of the Post-Industrial Economy. New York Times Book Review, 1987. 36 p.

16. Tinbergen J. The Duration of Development. Journal of Evolutionary Economics, 1995. Vol. 5(3). Pp. 333-39.

17. Triplett J.E. The Solow productivity paradox: What do computers do to productivity? The Canadian Journal of Economics. 1999. Vol. 32. №2. Pp. 310-334.

Статья поступила в редакиию 25.10.2021; одобрена после рецензирования 15.11.2021; принята к публикации 28.11.2021.

The article was submitted on 25.10.2021; approved after reviewing on 15.11.2021; accepted for publication on 28.11.2021.

\section{ИНФОРМАЦИЯ ОБ АВТОРАХ}

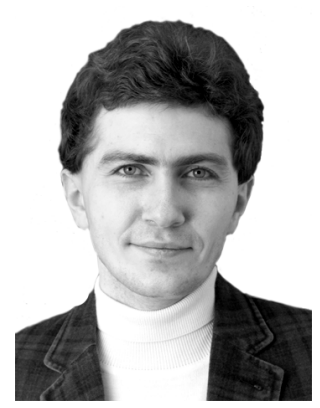

Сухарев Олег Сергеевич - доктор экономических наук, профессор, главный научный сотрудник Института экономики Российской академии наук.

Россия, г. Москва, Нахимовский пр., 32

Oleg S. Sukharev - Doctor of Economic Sciences, Professor, Chief Researcher, Institute of Economics, Russian Academy of Sciences.

32 Nakhimovskiy ln., Moscow, Russia 